BOOK REVIEW

\title{
Taylor, S.: THE PSYCHOLOGY OF PANDEMICS: PREPARING FOR THE NEXT GLOBAL OUTBREAK OF INFECTIOUS DISEASE, Cambridge Scholars Publishing, 2019, p. 158
}

\author{
Liliana Sabina ALECU1 \\ DOI: 10.35782/JCPP.2020.1.06
}

In hindsight, the writing was on the wall all along for the coronavirus crisis affecting the globe in 2020. While a rich scientific literature on the topic has been steadily developing from the 20 $0^{\text {th }}$ century onward, "The Psychology of Pandemics: Preparing for the Next Global Outbreak of Infectious Disease” (2019) by Steven Taylor can seem in this day and age, just like Steven Soderbergh's movie Contagion (2011), eerily prescient. All the more interesting, this is a book written not by an epidemiologist or a physician, but by a psychologist, combining a vast array of research on social psychology and human behavior with his considerable expertise on anxiety disorders. Accordingly, the aim of the book is to shed light on the psychological nature of pandemics, which usually tends to be overshadowed by the more obvious, medical problems which arise during epidemics.

One of the main arguments the book makes is that, although humankind has been making great strides from the earliest pandemics recorded in its history, about 10.000 years ago, the modern, interconnected "global village", to quote media theorist Marshall McLuhan (McLuhan, Powers, 1989), is not necessarily more immune to the serious existential threats epidemics pose. If in the century passed since the Spanish Flu of 1918, we've been making headway in developing vaccines for many illnesses, human psychology has been slow to adapt, meaning the risk of panic induced by modern media and social networks has never been higher. According to the author, our behaviour is instrumental in containing or spreading the pandemic. The anxiety factor can easily be ignored when dealing with an outbreak on a planetary scale, yet it is critical especially in the age of fake news, which can make people either underestimate or overestimate their potential of fighting the disease, leading them into all sorts of

${ }^{1}$ English Teacher, "St. Constantine and Helen” Elementary School, Bucharest, Romania; e-mail: alecu.lilis@gmail.com 
unhealthy practices or filling them with angst, which makes an easy prey for a deadly pandemic.

Here's a work of psychology masterly explaining how a myriad of factors, not only biological, but also cultural and social, determine the advancement of pandemics, from naming the disease (which can stereotype groups and sow divisions), to the prejudices we harbour, to the practices of traditional and Web 2.0 media (social media in particular), to our attitudes regarding vaccination, rumor spreading or the psychology of conspiracies - all of them, important factors in the puzzle of pandemics. These factors have clinical as well as emotional effects which are far-reaching. Consequently, knowing the whys and hows of people's behaviour during a pandemic is critical in managing a crisis and mitigating it, and of course preventing it in the future. As the author states in the preface of his elegantly rounded interdisciplinary work (drawing from disciplines like virology, epidemiology, sociology, public health), when disaster strikes, there are two fighting courses which reduce morbidity and mortality: vaccinations, when available, and - first and foremost - behavioral methods, from hand washing and proper hygiene to social distancing. One critical warning Taylor raises is that public health agencies have historically neglected the psychological factors an epidemic entails, leading to a meagre allocation of resources to this critical component of pandemics management. These range between experiencing high levels of distress, a worsening of pre-existing psychological problems, fearing for your health and the safety of your family, fearing for your job, the increase of stigmatization and xenophobia witnessed during pandemics, and so on.

As such, Taylor's declared goal is to fill an important goal in the literature on pandemics not only by outlining the psychological reactions to pandemics and reviewing the factors that come into play in spreading disease and emotional distress, but also by discussing empirical methods proven to address the said problems and describing the implications for public health policy.

The introductory chapter of the book provides the definition of a pandemic and a brief of the notable pandemics in world history. Taylor points out that the focus of the book is on influenza pandemics, as the most likely source of the next pandemic, while also reporting findings on other major outbreaks, such as SARS, Bubonic Plague or Ebola. Various predictions have been made on the nature of the next influenza pandemic, with the only certainty being that it is inevitable and serious (Laver \& Webster, 2001; Webby \& Webster, 2003). Taylor quotes Laver and Webster's assertion almost two decades ago that the world's population would have no immunity to this "new virus", whose spread would be helped by today's crowded conditions and modern rapid transportation facilities (p. 4). The stressors related to pandemics, taking their toll on regular people, are outlined, while the effects on the healthcare system and socioeconomic aspects of a pandemic are explored. The dynamics of the spread of pandemics are also detailed.

Chapter 2 is dedicated to contemporary methods for managing pandemics. There are four main methods used to manage the spread of infection: risk communication, which consists of educating the public on the dangers of an epidemic; pharmacological treatments, such as vaccines and antiviral therapies; hygiene practices; and social distancing, which is sometimes undermined by competing government interests (p. 21). 
The benefits and problems generated by these strategies are given proper attention and documented by means of historical examples.

Chapter 3 deals with the psychological reactions to pandemics, stating that emotional reactions to threats of harm, loss and change are common during pandemics. A sub chapter looks at the pursuit of quack remedies and dubious folk remedies, showing that during a pandemic people can be highly irrational in their decision-making. For example, when 2003's SARS outbreak occurred, there were many folk remedies, like using vinegar or spicy foods, all of which were ineffective (p. 28). Taylor also gives an account of the mass panic and civil unrest witnessed during pandemics, although prosocial behaviours are thought to be more common (p. 30). Attention is also given to the "sickness behaviour" by people who are infected, while the interaction between stress and the immune system is explored. Research suggests people prone to intense anxiety and negative emotions are more susceptible to pandemic influenza.

Chapter 4 takes into account personality traits as emotional vulnerability factors. Dr. Taylor reviews relevant traits that have been investigated in relation to infectious outbreaks (pp. 40-47). While high scores in traits like negative emotionality, overestimation of threat or intolerance of uncertainty mean people are more likely to be distressed by threatening events, like an influenza pandemic, coping strategies like monitoring and blunting have critical implications for both the individuals and the public health authorities' communication (p. 45). The author also discusses the so called unrealistic optimism bias, which can undermine prevention health behaviours, from the banal hand washing to seeking vaccination, and can even increase infection.

Chapter 5 is centered on the concept of "health anxiety" while exploring its implications in the context of a pandemic. For example, people with very low levels of health anxiety tend to neglect hygienic behaviours and to be nonadherent to social distancing (p. 50). At the other end of the spectrum, people with excessively high levels of health anxiety can misinterpret somatic stress reactions during pandemics and become functionally impaired, although they're physically healthy (p. 55). There's a clear relationship between beliefs about health and disease and health anxiety, which can be addressed via therapy.

Chapter 6 looks at the concept of "behavioral immune system", (BIS) providing insights into the social consequences of disease avoidance mechanisms. As part of the BIS, people become attentive to cues that indicate pathogens, therefore reacting through disgust, fear and the urge to escape (p. 57). Of particular note is the section dedicated to how BIS triggers stigmatization and xenophobia. During Bubonic Plague in Italy, in 1576, in Milan, homes of Jews were raided because of the prejudices related to them, which saw them as sources of contagion (p. 60). Also, labelling pandemics a certain way ("Asian flu" or "Chinese virus", for example) can have severe psychological implications and amplify discrimination during pandemics (p. 62).

Chapter 7 dissects how conspiracy theories are set in motion during a pandemic, concerning both the cause of infection but also the vaccines. Conspiracy theories are attempts to explain the causes of significant events by claiming they are due to secret plots by powerful actors (Douglas, Sutton, \& Cichocka, 2017). Taylor notes that 
conspiracy theories appear to be a culturally universal phenomenon (p. 63), and then provides historical evidence. Methods for reducing conspiratorial thinking are subsequently proposed, hard as they may be to implement (p. 67). Chapter 8 explores several social psychological factors, like beliefs and fears, and their circulation within social networks. The contagion with rumors receives particular attention within the larger, medical contagion. If unchecked, rumors can heave disruptive social effects and lead to widespread fear, hostility and suspicion (p. 70). How news media and social media reflect pandemics and the ensuing role in the spread of misinformation and fear is also highlighted, providing critical insights into the making of a crisis (pp. 72-77).

Chapter 9 offers strategies and recommendations for improving risk communication, a vital part of the response to pandemics, as nonadherence to advice from health authorities is a widespread problem (p. 79). Different styles of communication work for different people: some are motivated by fear, while for others cultural sensitivity is a better approach (p. 85).

Chapter 10 addresses a sensitive topic of our times, namely vaccination, which is important, if not vital, in containing the next influenza pandemic (p. 87). Taylor evokes the role of negative beliefs about vaccination in pandemics, citing several outbreaks of measles which were caused by parental anti-vaccination beliefs. The author also asks if mandatory vaccination is a viable option, given the never-ending public debate on the issue. Segmentation based on where people work and with whom they come into contact (e.g., healthcare workers) may prove the best option (p. 96).

Chapter 11 looks at ways to treat pandemic-related emotional distress, commonly developed during such stressing times when many people are sick and staff shortages are common. Several screening methods are investigated and valuable stress management advice for the general public is provided (pp. 104-105), while keeping healthcare workers and their stressors in mind ("helping the helpers").

Chapter 12, concluding the book, attempts to draw the portrait of the next major pandemic (which, bear in mind, would hit the world only a year after the book's publishing, testament to its relevance). It predicts the problems posed by the pandemic will be manifold and will highly test the resilience of both individuals and the State (pp. 107-109). It also outlines a roadmap for future research in the field.

"The Psychology of Pandemics: Preparing for the Next Global Outbreak of Infectious Disease" by Steven Taylor (2019, Cambridge Scholars Publishing) makes for easy and eye-opening reading. The amount of information it packs in its 111 pages is truly remarkable. While certain topics would have certainly deserved to be expanded, the book is a salutary contribution to the fields of social psychology and epidemiology. A must read for public health officials, but also for the general public, all the more in the COVID-19 reality. 
Book review | 101

\section{References}

Laver, G., \& Webster, R.G. (2001). Introduction. Philosophical Transactions of the Royal Society B: Biological Sciences, 356, 1813-1815.

Webby, R.J., \& Webster, R.G. (2003). Are we ready for pandemic influenza? Science, 302, 15191522.

Douglas, K.M., Sutton, R.M., \& Cichocka, A. (2017). The psychology of conspiracy theories. Current Directions in Psychological Science, 26(6), 538-542. doi:10.1177 /0963 721417718261

McLuhan, M., \& Powers, B. R. (1989). The global village: Transformations in world life and media in the 21 st century. Communication and Society. 